PROCEEDINGS OF THE

AMERICAN MATHEMATICAL SOCIETY

Volume 29, Number 3, August 1971

\title{
A CHARACTERIZATION OF REGULARITY IN TOPOLOGY
}

\section{OSWALD WYLER ${ }^{1}$}

ABSTRACT. We show in this paper that a topological space satisfies $T_{3}$ (which we do not intend to imply $T_{2}$ ) if and only if convergence of filters is a continuous relation. In particular, a Hausdorff space is regular if and only if convergence of filters is a continuous mapping. We propose a new, categorically motivated, definition of continuous relations between topological spaces, and we compare it with two existing continuity concepts for relations.

Let $(E, \tau)$ be a topological space. We denote by $E^{*}$ the set of all filters on $E$ which converge for $\tau$ to some point of $E$. For $X \subset E$, we put $X^{*}=\left\{\varphi \in E^{*}: X \in \varphi\right\}$. Then $\varnothing^{*}=\varnothing$ for the empty set, and

$$
(X \cap Y)^{*}=X^{*} \cap Y^{*}, \quad \dot{x} \in X^{*} \Leftrightarrow x \in X,
$$

for subsets $X, Y$ of $E, x \in E$, and $\dot{x}=\{X \subset E: x \in X\}$. We regard convergence of filters for $\tau$ as a relation $q: E^{*} \rightarrow E$, writing $\varphi q x$ if $\varphi$ converges to $x$. This relation is a mapping if and only if $(E, \tau)$ is a Hausdorff space. For $X \subset E$, we have $q\left(X^{*}\right)=\bar{X}$, the closure of $X$ for $\tau$.

It seems natural to impose a topology on $E^{*}$ by using the sets $U^{*}$, with $U$ open for $\tau$, as a basis of open sets. The preceding paragraph shows that this works, and we denote the topology of $E^{*}$ thus defined by $\tau^{*}$. With this notation, we state the following theorem.

TheOREM 1. A Hausdorff space $(E, \tau)$ is regular if and only if convergence of filters on $E$ for $\tau$ defines a continuous map $q:\left(E^{*}, \tau^{*}\right) \rightarrow(E, \tau)$.

Instead of proving Theorem 1 directly, we generalize it. Theorem 1 is an immediate corollary of Theorem 3 below. We need some definitions.

Let $r: E \rightarrow F$ be a relation between two sets. For $X \subset E$ and $Y \subset F$, we put $y \in r(X)$ if $y \in F$ and $x r y$ for some $x \in X$, and $x \in r^{-1}(Y)$ if $x \in E$ and $x r y$ for some $y \in Y$. One sees easily that

$$
r(X) \cap Y=\varnothing \Leftrightarrow X \cap r^{-1}(Y)=\varnothing .
$$

If $E$ and $F$ are topological spaces, then $r$ is called upper semicontinuous

Received by the editors June 12, 1970 and, in revised form, October 15, 1970.

AMS 1970 subject classifications. Primary 54D10; Secondary 18B10, 54C05.

Key words and phrases. Regular topological space, regular Hausdorff space, convergence of filters, continuous relation, upper semicontinuous relation, lower semicontinuous relation.

1 Research supported in part by NSF Grant GP-8773.

Copyright (C) 1971, American Mathematical Society 
if $r^{-1}(Y)$ is closed in $E$ for every closed $Y \subset F$, and $r$ is called lower semicontinuous if $r^{-1}(Y)$ is open in $E$ for every open $Y \subset F$. These concepts have been used by various authors; see e.g. [1, Chapter VI] or [3].

A relation $r: E \rightarrow F$ between topological spaces has been called continuous if $r$ is both upper and lower semicontinuous. We propose a different definition. We call $r: E \rightarrow F$ continuous if, for a topological space $A$ and mappings $f: A \rightarrow E$ and $g: A \rightarrow F$ such that $f(u) r g(u)$ for all $u \in A$, continuity of $f$ always implies continuity of $g$.

This can be simplified. Let $R \subset E \times F$ be the graph of $r$ and $f_{1}: R \rightarrow E$ and $g_{1}: R \rightarrow F$ the projections, i.e. $f_{1}(x, y)=x$ and $g_{1}(x, y)=y$ if $x r y$. Provide $R$ with the coarsest topology for which $f_{1}$ is continuous. If $r$ is continuous, then $g_{1}$ is continuous for this topology of $R$. In fact, this is not only necessary but also sufficient for continuity of $r$. For if $f: A \rightarrow E$ and $g: A \rightarrow F$ are mappings such that $f(u) r g(u)$ for every $u \in A$, then $f=f_{1} h$ and $g=g_{1} h$ for a unique mapping $h: A \rightarrow R$, and $h$ is continuous, for the given coarse topology of $R$, if $f$ is continuous. Thus continuity of $f$ implies continuity of $g$ if $g_{1}$ is continuous.

We shall study continuous relations elsewhere in greater detail and in a more general setting. We mention here only that all three continuity concepts defined above coincide with the usual continuity if $r$ is a mapping, and we connect continuity with upper and lower semicontinuity by the following result.

Theorem 2. A continuous relation $r: E \rightarrow F$ between topological spaces is upper semicontinuous if and only if its domain $r^{-1}(F)$ is closed in $E$, and $r$ is lower semicontinuous if and only if $r^{-1}(F)$ is open in $E$.

Proof. If $r$ is upper semicontinuous, then $r^{-1}(F)$ is closed in $E$. Conversely, let $R \subset E \times F$ be the graph of $r$ and $f_{1}: R \rightarrow E$ and $g_{1}: R \rightarrow F$ the projections, as above. Provide $R$ with the coarsest topology for which $f_{1}$ is continuous, with the sets $f_{1}^{-1}(X), X$ closed in $E$, as closed sets. If $r$ is continuous and $Y$ closed in $F$, then $g_{1}^{-1}(Y)$ is closed in $R$, and thus $g_{1}^{-1}(Y)=f_{1}^{-1}(X)$ for a closed set $X \subset E$. One sees easily that $r^{-1}(Y)=X \cap r^{-1}(F)$ in this situation. Thus $r^{-1}(Y)$ is closed if $r^{-1}(F)$ is closed. The same argument, with closed sets replaced by open sets, shows that $r$ is lower semicontinuous if and only if $r^{-1}(F)$ is open.

The following example shows that Theorem 2 has no obvious converse. For every topological space $E$, the full relation $r: E \rightarrow E$ with graph $E \times E$ is both upper and lower semicontinuous. On the other hand, we have $f(u) r g(u)$ for all $u \in A$ if $f: A \rightarrow E$ and $g: A \rightarrow E$ are arbitrary mappings. Thus $r$ is continuous only if $E$ is an indiscrete space. 
We need one of the separation axioms introduced by Davis [2]. Davis calls a topological space $(E, \tau)$, with filter convergence $q$, an $R_{\mathbf{0}}$ space if always $\dot{x} q y \Rightarrow \dot{y} q x$ for $x, y$ in $E$. It is shown in [2] that $T_{1}$ is equivalent to the conjunction of $T_{0}$ and $R_{0}$, and that $T_{3}$ (called $R_{2}$ in [2]) always implies $R_{0}$.

THEOREM 3. The following three statements are logically equivalent for a topological space $(E, \tau)$ with filter convergence $q$.

(i) $(E, \tau)$ is a $T_{3}$ space.

(ii) $q:\left(E^{*}, \tau^{*}\right) \rightarrow(E, \tau)$ is continuous.

(iii) $(E, \tau)$ is an $R_{0}$ space and $q$ is upper semicontinuous.

Proof. Assume first $T_{3}$ and consider $f: A \rightarrow E^{*}$ and $g: A \rightarrow E$ with $f$ continuous and $f(u)$ converging to $g(u)$ for all $u \in A$. If $U$ is open in $E$ and $g(u) \in U$, then $g(u) \in V$ and $\bar{V} \subset U$ for some open $V$. For this $V$, we have $V \in f(u)$, and $V \in f(v)$ implies $g(c) \in \bar{V}$. Thus $u \in f^{-1}\left(V^{*}\right)$ and $f^{-1}\left(V^{*}\right) \subset g^{-1}(U)$. This shows that $g^{-1}(U)$ is open, and hence $g$ continuous.

If $q$ is continuous, then $q$ is upper semicontinuous by Theorem 2. If $\dot{x} q y$, let $A$ be the space with two points $u, v$, and with $\{v\}$ open, but not closed. Put $f(u)=f(v)=\dot{x}$ and $g(u)=x, g(v)=y$. Then $f$ is continuous, and $f(z) q g(z)$ for $z \in A$. Thus $g$ is continuous. If $V$ is open and $x \in V$, then $g^{-1}(V)$ is open and $u \in g^{-1}(V)$. Thus $g^{-1}(V)=A$, and $y \in V$. This shows that also $\dot{y} q x$, and $E$ is $R_{0}$.

Assume now (iii), and let $F$ be closed in $E$ and $x \in E \backslash F$. If $\dot{x} q y$, then $\dot{y} q x$, and $y \in F$ would imply $x \in \bar{F}=F$. Thus $\dot{x} \notin q^{-1}(F)$. It follows that $\dot{x} \in V^{*}$ for an open set $V$ with $V^{*} \cap q^{-1}(F)=\varnothing$. But then $x \in V$, and $\bar{V} \cap F=q\left(V^{*}\right) \cap F=\varnothing$. Thus $E$ satisfies $T_{3}$.

The following example shows that $R_{0}$ cannot be omitted from Theorem 3. The space with two points and three open sets (used in the proof of the theorem) is $T_{0}$ but not $T_{1}$, and hence a fortiori not $T_{3}$ or $R_{0}$. But one sees easily that $q$ is upper semicontinuous for this space.

REMARK. All results of this note remain valid if $E^{*}$ is replaced by a set of convergent filters which contains all convergent ultrafilters.

\section{REFERENCES}

1. C. Berge, Topological spaces, Macmillan, New York, 1963.

2. A. S. Davis, Indexed systems of neighborhoods for general topological spaces, Amer. Math. Monthly 68 (1961), 886-893. MR 35 \#4869.

3. E. A. Michael, Topologies on spaces of subsets, Trans. Amer. Math. Soc. 71 (1951), 152-182. MR 13, 54.

Carnegie-Mellon University, Pittsburgh, Pennsylvania 15213 